\title{
STOCHASTIC BEHAVIOR STUDY OF BACKOFF ALGORITHMS IN CASE OF HIDDEN TERMINALS
}

\author{
Tahiry RAZAFINDRALAMBO*, Fabrice VALOIS \\ CITI laboratory, INRIA ARES team, INSA Lyon \\ 21, Avenue Jean Capelle - 69621 Villeurbanne Cedex - France \\ firstname. lastnamedinsa-lyon.fr
}

\section{ABSTRACT}

In MANET, the hidden terminals problem is a common situation which leads to poor performances due to collisions and unfairness behavior of nodes. The use of RTS/CTS mechanism is helpful to improve the performances but not sufficient: it is important to design new backoff algorithms. But, before, it is necessary to understand how backoff algorithms deal with longterm and short-term unfairness and also with performances. In this paper, based on a stochastic process algebra, we provide a new study of the hidden terminals problem. The Binary Exponential Backoff and the Double Increase Double Decrease backoff algorithms are studied in depth in this case. The state of the medium (idle, occupied by either collision or transmission) is computed and the probability of the different backoff's stage is analysed. The short-term unfairness is clearly shown with a new metric. All results are given in both basic access and RTS/CTS one.

\section{INTRODUCTION}

Since the last decade, MANET have been widely studied, mainly in the point of view of routing protocols. In order to provide performance study, simulation tools was used to compute delay, mobility robustness, etc. More recently, testbeds was deployed to show their feasibility or to validate distributed algorithms. Such practical experiments was based on 802.11 wLAN protocol [5] and the main results was twice. Firstly, 802.11 exhibits an unfair behavior and secondly, there exist different scenarios where the 802.11 MAC protocol leads to poor performances. Because of the instability of the radio environment, practical experiments are not suitable to the study of both fairness and performance evaluation. In this paper, we propose an analytical model to investigate the key scenario of hidden terminals using 802.11. A complete study of it behavior is given. Because it is also necessary to provide a generic approach in order to re-use such model for another scenario, we use a stochastic process algebra. This compositional approach is suitable for network performance evaluation and allow to provide generic model which can be extended easily.

The paper is organized as follows. After the related works, a general overview of the process algebra PEPA is given in section III.. The section IV. is focused on the analytical model. Numerical results are discussed in section V., followed by a conclusion and future works.

\footnotetext{
* financed by France Telecom R\&D under CRE-46128746
}

\section{RELATED WORKS}

Two main analytical approaches deal with the study of 802.11 DCF mode. The first one is based on the performance analysis especially the binary exponential backoff (BEB) [1] and its derivation [2], whereas the second one focuses on short term or long term fairness analysis $[4,9]$.

[1] proposed an analytical study of 802.11 DCF mode based on a Markov chain modeling a node. However this study is carried out on a single hop network. This analysis provides an accurate performance analysis of the BEB algorithm but no fairness analysis of the backoff algorithm is given. Because this model deals with infrastructure networks, it is not suitable to study multi-hop networks. Based on the Markov model of [1], [2] studies the DIDD (Double Increase and Double Decrease) backoff algorithm. DIDD is compared to the classical BEB only in the case of single hop networks. No analysis on multi hop networks have been done and no modification of the model have been made to study fairness behavior of these two models. Very few works are focused on multi-hop networks using 802.11 DCF. In [4] an analytical model of the three pairs scenario is studied [3]. This model is based on a Markov chain with an accurate model of BEB. As far as we know this is the only paper that deals with both the performance analysis of the BEB algorithm and fairness in 802.11 multi-hop networks. This model provides an accurate model of the three pairs scenario with the BEB that make the long term unfairness appears. Nevertheless, this model can not be used to study another scenarios. [6,9] design an analytical Markov model (close to each other) to evaluate the short term unfairness of 802.11 considering the hidden terminal scenario. [9] do not show performance evaluation of 802.11 but only the unfairness behavior of 802.11 using basic and RTS/CTS mechanism. This work is very close to ours from the scenario point of view as we both deal with the hidden terminal problem. The difference is that in our work we study the performance of the 802.11 , the behavior of the backoff algorithm in addition to the unfairness analysis. We also extend our analysis to DIDD algorithm.

\section{A GENERAL OVERVIEW OF A STOCHASTIC PROCESS ALGEBRA: PEPA}

\section{A. The formalism syntax}

A system is viewed as a set of components which carry out activities. Each activity $(\alpha, r)$ is characterized by an action type $\alpha$ and an activity rate $r$ which is exponentially distributed. Because of the exponential distribution of the activity duration, the underlying Markov process of a PEPA model is a continu- 
ous time Markov chain [7]. PEPA formalism provides a set of combinators which allows expressions to be built, defining the behavior of components. Below, we introduce only the combinators which are necessary to our model. For more details about the formalism, see [7].

Constant: $S \stackrel{\text { def }}{=} P$ It allows to assign names and behavior to components. To component $S$, the behavior of component $P$ is assigned.

Prefix: $S \stackrel{\text { def }}{=}(\alpha, r) . P$ After $S$ has carried out the activity $(\alpha, r)$, it will behave as component $P$. In the case of cooperation or shared activities, the activity rate of this action is outside the control of this component and is determined by another component. Such actions are carried out jointly with another component. The activity rate is denoted $T$.

Choice: $P_{1}+P_{2}$ It models competition between components. The system may behave either as component $P_{1}$ or as $P_{2}$. All current activities of the components are enabled. The first activity to complete distinguishes one of these components, the other is discarded.

Cooperation: $P \bowtie Q$ It allows the synchronization of components $P_{1}$ and $P_{2}$ over the activities in the cooperation set $L$. Components may proceed independently with activities whose types do not belong to this set. In a cooperation, the rate of a shared activity is defined as the rate of the slowest component. The rate of an activity may be unspecified for a component $(\top)$ : the rate of this shared activity is defined by the other component in cooperation.

\section{B. The Markov Process}

In a PEPA model, when a component $P$ carries out an activity $(\alpha, r)$ and subsequently behaves as component $P^{\prime}, P^{\prime}$ is said to be a derivative of $P$. From any PEPA component $P$, the derivative set $(d s(P))$, is the set of derivatives (behaviors) which can evolve from the component. This set is defined recursively. From the derivative set, we can construct the derivation graph. The derivation graph is a directed multi-graph whose set of nodes is $d s(P)$ and whose arcs represent the possible transitions between them. The underlying Markov process of a PEPA model is a continuous time Markov process. The generation of this process is based on the derivation graph of the model. A state is associated with each graph node and the transitions between states are derived from the arcs of the graph.

\section{Solving the Markov chain}

PEPA is supported by an experimental tool called PEPA Workbench. From a description of a PEPA model, this tool provides the stationary behavior (probability distribution) of the modeled system.

\section{HIDDEN TERMINALS MODELLING}

In the hidden nodes problem (figure 1), we consider two transmitters with only one receiver such as the transmitters are fully independent: if it send data simultaneously, it leads to several collisions at the node 2. Based on the PEPA paradigm, four components are used. TR_O (resp. TR_l) is associated

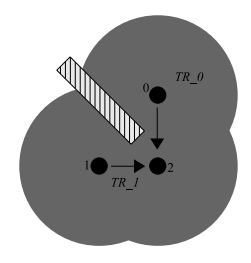

Figure 1: Hidden nodes configuration

to the transmitter-receiver $\{0,2\}$ (resp. $\{1,2\}$ ). A component $B O_{-} i$ is used to model the backoff algorithm of the node $i, i \in\{0,1\}$. Because of the compositional approach of PEPA, it is easy to model another backoff algorithm. The last component deals with the medium modelling. In fact, this component models the nodes interactions through the use of the radio channel. Based on the medium modelling, it is also possible to study different scenario as it is mentioned in [8]. Next, we describe each components and discuss about the assumptions made.

\section{A. The transmitter-receiver model}

As the two transmitters-receivers exhibit the same behaviour due to the use of the same MAC protocol, the components modelling the two pairs are the same. Let denoted $T R \_i_{-} 000_{i \in\{0,1\}}$ the component which modelled the transmitter-receiver $\{i, 2\}$.

$$
\begin{aligned}
& T R_{-} i_{-} 000 \stackrel{\text { def }}{=} \quad\left(D I F S, \mu_{-} \text {difs }\right) . T R_{-} i_{-} 001 \\
& T R_{-} i_{-} 001 \stackrel{\text { def }}{=} \quad\left(D B_{-} i, T\right) . T R_{-} i_{-} 002 \text {; } \\
& T R_{-} i_{-} 002 \stackrel{\text { def }}{=} \quad\left(\text { send, } \mu_{-} \text {trans }\right) . T R_{-} i_{-} 003 \\
& T R_{-} i_{-} 003 \stackrel{\text { def }}{=} \quad(\text { Ack_i } i, T) \cdot T R_{-} i_{-} 004+(\text { collision, } T) \cdot T R_{-} i_{-} 005 \\
& T R_{-} i_{-} 004 \stackrel{\text { def }}{=} \quad\left(\text { succ_i, } \mu_{-} \text {trans }\right) . T R_{-} i_{-} 006 \\
& T R_{-} i_{-} 005 \stackrel{\text { def }}{=} \quad\left(\text { coll }{ }_{-} i, \mu_{-} \text {trans }\right) . T R_{-} i_{-} 000 \\
& T R_{-} i_{-} 006 \stackrel{\text { def }}{=}\left(\text { sync_i, } \mu_{-} \text {trans }\right) . T R_{-} i_{-} 000
\end{aligned}
$$

Eight actions are used in order to model the behaviour of CSMA/CA. Before to send a packet, the transmitter $i$ decreases its DIFS counter with the activity $\left(D I F S, \mu_{-} d i f s\right)$. Because PEPA is a stochastic paradigm, the rate of action type DIFS should follow an exponential distribution but, in the point of view of 802.11, the DIFS counter follow a static distribution $(D I F S=50 \mu s)$. More generally, in this work, all static duration associated to inter-frame spacing or backoff slot-time are modelled with an exponential distribution. In this scenario, the transmitter $i$ always see the channel as free ${ }^{1}$, so, the draw backoff is computed. The activity $\left(D B_{-} i, \top\right)$ is shared with the component $B O_{-} i_{-} x$ modelling the backoff algorithm. The rate associated to $T$ will be computed in $B_{-} i_{-} x$ in function of the number of collisions the current transmission have undergone. After the backoff decreasing, the transmitter $i$ send its packet and wait for an acknowledgement or a collision. Such information come from the medium. Because it is shared by both the transmitters, only the component Medium knows if there is a collision or a successful transmission. The

\footnotetext{
${ }^{1}$ we do not consider interruption due to the ACK of the other node because it is negligible and simplifies the model (see model validation)
} 
two concurrencies activities $($ Ack, $\top$ ) and (collision, $\top$ ) are finally shared with Medium. The activities coll_$_{-} i$ and $\operatorname{succ} \__{-} i$ provide the synchronisation with the backoff component $B O \_i \_x$. The activity sync_i allows to compute the number of successive successful transmissions with the Medium.

\section{B. The backoff model}

The component $\mathrm{BO}_{-} i_{-}$is associated to the node $\mathrm{i}, i \in\{0,1\}$, and models the binary exponential backoff (BEB) algorithm used in 802.11. 8 transmissions attempts are allowed. The rate of the activity $D B_{-} i$, called $f_{-} j-j \in\{0,5\}$, depends on the number of consecutive collisions the current transmission have undergone. Because of the memory-less property of the exponential distribution, the mean of $f_{-j}$ is the mean duration time of the contention window with $j$ collisions. It means that $f_{-} l$ is the mean duration time of the backoff before the first transmission. $f_{-} l$ is associated to the initial contention window $\left[0 . .2^{5}\right]$. Finally, $f_{-} l$ is equal to $20 \mu s \times\left(2^{5}-1\right) / 2$. More generally, $\forall i \in\{0 . .5\}, f_{-} i$ is the mean duration time of the contention window $\left[0 . .2^{5+i}\right]$.

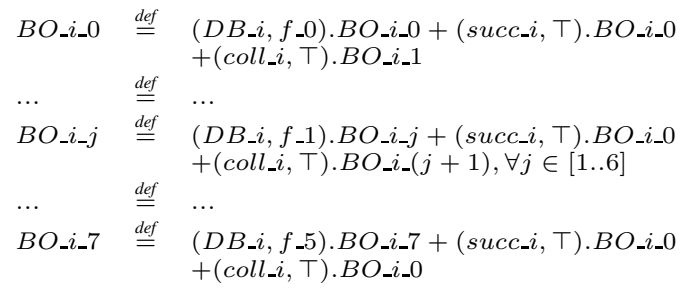

We said this approach is generic because it is possible to model another backoff algorithm like DIDD and study the behavior of this algorithm in the case of the current scenario without any modification in the others components.

\section{The medium}

All the activities of this component are shared activities with the transmitter-receiver $\{0,2\}$ and $\{1,2\}$ except for the frag activity. The component Medium is associated to the idle state of the medium. The components Medium_00_x $\forall x \in[1 \ldots 10]$ are associated to the transmission of a packet. During the transmission, a collision can occur if the other transmitter also sends a packet: the transmission can be interrupted. Because a PEPA activity is an atomic process, it is necessary to model such interruption. The local activity frag models the transmission of a part of the packet. This activity is in concurrency with the shared activity (send, $\top$ ) modelling the use of the medium by the other transmitter. It leads to the components Medium_O0_14 and Medium_00_13 which modelled the collision. The rate $\mu_{\text {_collision }}$ of the collision action is equal to the rate of a successful transmission because it leads to the same channel occupancy.

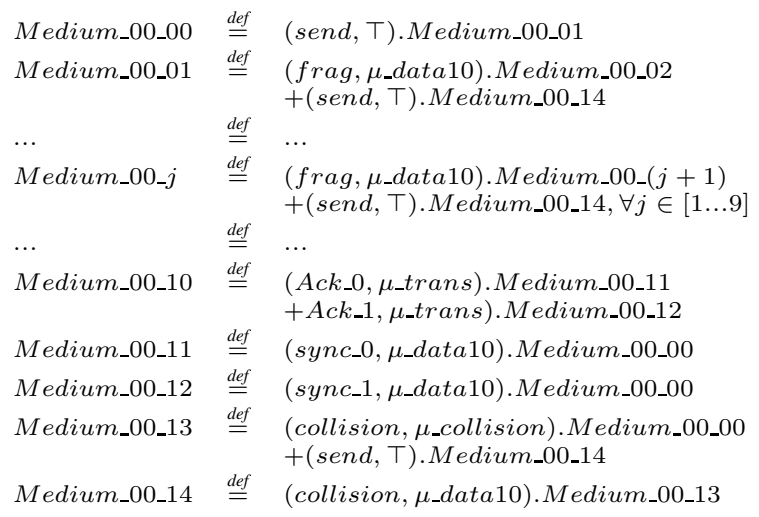

It is possible to modify the model of the medium in order to study the 3-pairs scenario [4] or any another MANET configuration. Such property leads to a model which is very useful to study different MANET topologies.

In order to compute the number of successful successive transmissions for one node the medium model has been modified. The medium component can be seen as Medium $i_{-} j$ where $i$ is the counter. If no collision and no interruption by a successful transmission of the other node occurred $i$ is incremented.

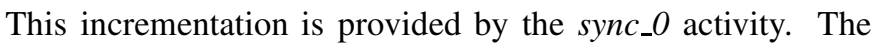
new medium model is represented below.

\begin{tabular}{|c|c|c|}
\hline $\begin{array}{l}\text { Medium_( }(i-1)_{-} j \\
\ldots\end{array}$ & $\begin{array}{l}\stackrel{\text { def }}{=} \\
\stackrel{\text { def }}{=}\end{array}$ & $\begin{array}{l}\text { for } j=0 \ldots 14 \\
\ldots\end{array}$ \\
\hline Medium_i_j & $\stackrel{\text { def }}{=}$ & remain the same $\forall j \in[1 \ldots 10]$ \\
\hline Medium_i_11 & $\stackrel{\text { def }}{=}$ & $\left(\right.$ sync_ $0, \mu_{-}$data 10$) \cdot$ Medium $(i+1) \_00$ \\
\hline Medium_i_12 & $\stackrel{\text { def }}{=}$ & $\left(\right.$ sync_1, $\left.\mu_{-} d a t a 10\right)$. Medium_00_00 \\
\hline Medium_i_13 & $\stackrel{\text { def }}{=}$ & $\begin{array}{l}\left(\text { collision, } \mu_{-} \text {collision }\right) . M e d i u m_{-} 00 \_00 \\
+(\text { send }, \top) . M e d i u m_{-} i_{-} 14\end{array}$ \\
\hline Medium_i_14 & $\stackrel{\text { def }}{=}$ & (collision, $\mu_{-}$data10).Medium_i_13 \\
\hline $\begin{array}{l}\text { Medium }_{-}(i+1)_{-} j \\
\ldots\end{array}$ & $\begin{array}{l}\stackrel{\text { def }}{=} \\
\stackrel{\text { def }}{=}\end{array}$ & for $j=0 \ldots 14$ \\
\hline
\end{tabular}

\section{A general model for hidden nodes}

The model component Scenario is viewed as the interaction between the two transmitters, the receiver and the medium sharing.

$$
\begin{aligned}
& \text { Scenario } \stackrel{\text { def }}{=} \quad\left(\left(T R \_0 \_000 \coprod_{K} B O_{-} 0 \_0\right) \|\right. \\
& (T R \_1 \_000 \underbrace{}_{L} \text { BO_1_0) } \bigotimes_{M} \text { Medium_00_00 }
\end{aligned}
$$

The cooperation sets are defined as: $K=$

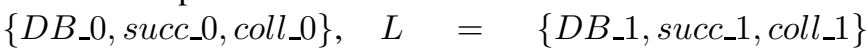
and $M=\{$ send,ack_0,ack_1, sync_0, sync_1, collision $\}$.

\section{E. Improved model with RTS/CTS mechanism}

This model can be extended in order to model RTS/CTS. Basically, we complete the model with a semaphore model when a node has a packet to send. Two activities start and stop are used to lock the channel before to transmit and to release it after a successful transmission. In appendix, we give the transmitter model for the node 0 , the medium model and the whole model. 


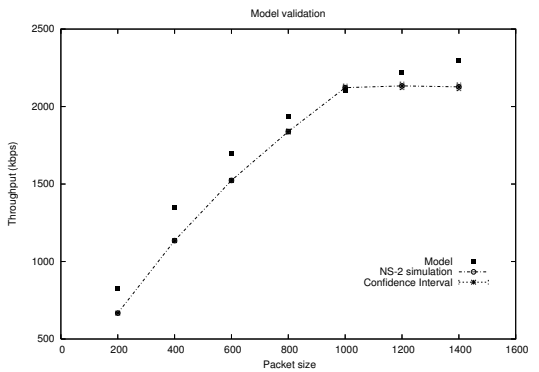

Figure 2: Model validation (11Mbps)

\section{PERFORMANCES ISSUES}

In order to solve this model, we use PEPA Workbench and, due to the large space states, the powerful PRISM tool' ${ }^{2}$. Based on the analytical model described above, we provide numerical results. These results are compared to a simulation model based on ns-2. We have computed:

- the throughput for each transmitter,

- the medium state distribution: the probability of a free medium/successful transmission/collision,

- the backoff stage distribution;

- the probability the $i^{\text {th }}$ transmission is successful while the $[1 . .(i-1)]^{t h}$ were successful and not stopped by another transmission.

All the results are computed for the basic access mechanism and the RTS/CTS with different data rates and packets length. But, due to the lack of place, only a data rate of $11 \mathrm{Mbit} / \mathrm{s}$ and 1000 bytes data (if not specified) is considered and the simulation results are given in order to validate our model.

Throughput of each transmitter :The throughput computed by simulation and using the analytical model described above is shown figure 2. This result is useful to validate how relevant our model is: both results are close. The others comments for this figure are some well-known properties: using a data rate of $11 \mathrm{Mbit} / \mathrm{s}$, the useful throughput is less than 2.2Mbit/s depending of the packet size.

Medium state distribution :We study here how the medium is used. The component Medium_i_OO models the idle state, the components Medium_i_14 and Medium_i_13 deal with the medium occupied by collisions while the others components model successful transmission. Based on the steady state, we can compute the different probabilities for each medium state. Fig. 3,4 and 5 provide the results for both the basic access mechanism and RTS/CTS for BEB and DIDD. When RTS/CTS is used, the collision probability is independent of the packet size and the data rate. Clearly, the RTS/CTS is very efficient because the collision probability is more important in the case of the basic access mechanism. The RTS/CTS is also more efficient in the point of view of successful transmission. Nevertheless, we note that the RTS/CTS sequence reduce the packet pro-

\footnotetext{
${ }^{2}$ http://www.cs.bham.ac.uk/ dxp/prism/
}

portion payload, there is here a trade off to find. Finally, whatever the access mechanism is, the probability of idle medium decreases when the packet size increases. Simultaneously, the probability of collision increases in the case of the basic access mechanism. We can notice that DIDD is more efficient than BEB from medium usage point of view and, for both DIDD and BEB using basic access mechanisms, there exists an optimal packet size that is different to the maximum packet size as in single hop network.

Backoff stage distribution :Fig. 6 illustrates the backoff stage distribution. Such probabilities are computed through the value of the steady-state associated to the components $B O_{-} i$ ( $i \in\{1,2\}$ ). More precisely, $B O_{-} i_{-} O$ models the backoff stage linked to the initial contention window while $B O_{-} i_{-} j$ models the new contention window after $j$ collisions. Except for the initial contention window, it is important to under-light that the backoff stage distribution is not very sensitive to the access mechanism (basic or RTS/CTS). With BEB there are 7 backoff stages (retry limit) that does not exists in DIDD. Note that DIDD backoff distribution is higher for stage 0 and 5 . We can deduce from this figure that when using DIDD, a node can have two distincts behavior: when the station access the medium, is backoff stage stay close to 0 , and when it encounters collision its backoff stage is close to 5. In BEB this behavior does not appear because even with one successful transmission the backoff stage is set to 0 and also because of the retry limit. This can be interpret as an unfair behavior of the DIDD algorithm.

Probability for the $i^{\text {th }}$ transmission to be successful while the $[1 . .(i-1)]^{\text {th }}$ were successful and not stopped :Fig. 7 shows the $\alpha_{i}, i \in[1 . .11]$, where $\alpha_{i}$ is the probability the $i^{t h}$ transmission is successful while the $[1 . .(i-1)]^{\text {th }}$ were successful and not stopped by another transmission. $\alpha_{i}$ is computed as follow :

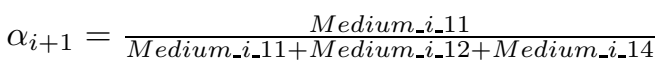

$\alpha_{i}$ illustrates the short-term unfairness. The two algorithms and the two access mechanisms appear to be unfair because, $\alpha_{i}$ is a monotonic increasing function. More the transmitter sends successful successive transmissions, more the next transmission has a chance to succeed. DIDD is unfair compared to BEB for both basic access and for RTS/CTS access because $\alpha_{i}$ is greater for DIDD than for BEB $(\forall i)$. We can also see that RTS/CTS access is unfair compared to basic access. For BEB this is true for more than 6 successive successful transmissions.

Fig. 8, shows the probability of being stopped either by a collision or by another transmission during a series of successive transmissions. From this figure we can see that : 1) for basic access mechanisms the probability to be stopped by another node transmission are the same and are very low for both algorithms. The probability of collision is lower for DIDD than for BEB and this confirms the fact that DIDD is unfair compared to BEB. The constant value of the DIDD collision probability is due to the stability of the algorithms that always remain close to the same value 2) for RTS/CTS access, collision probability is lower than interruption probability for both algorithms but are not close to 0 this is due to the small size of RTS packets. Here we also see that DIDD is unfair compared to BEB 

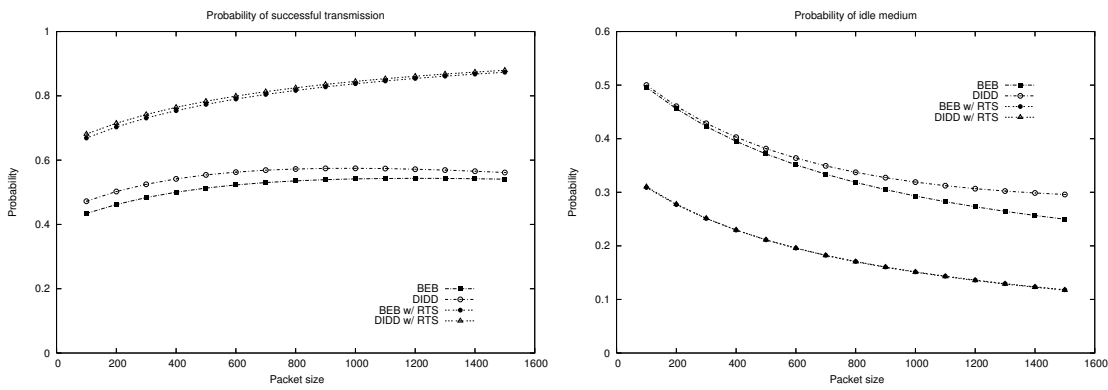

Figure 3: Success probability

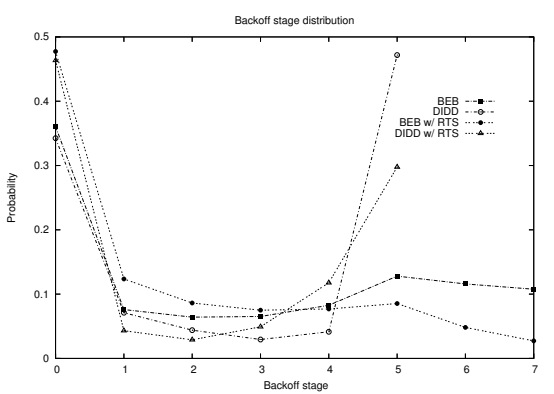

Figure 6: Backoff distribution
Figure 4: Idle probability

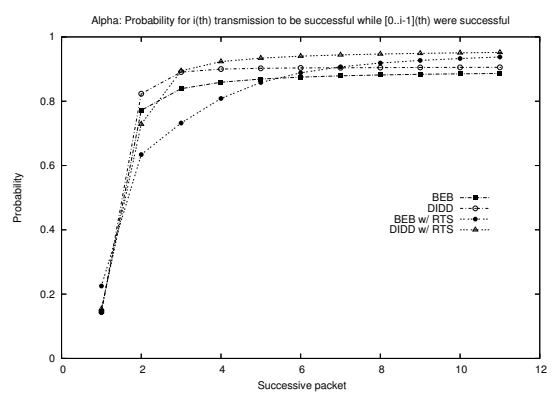

Figure 7: $\alpha_{i}$

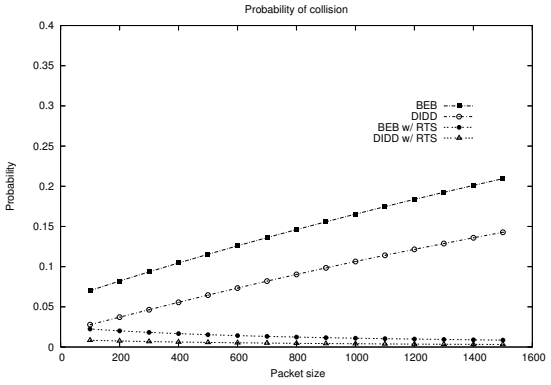

Figure 5: Collision probability

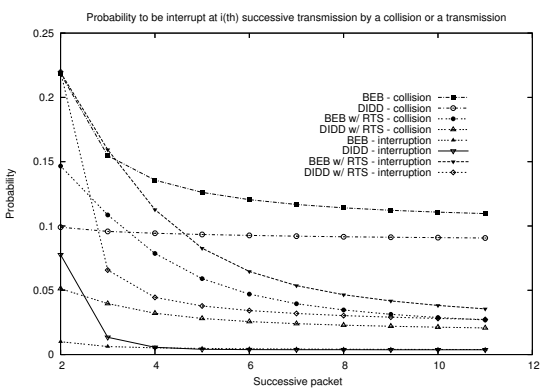

Figure 8: Probability for the $i^{\text {th }}$ transmission to collide or to be stopped because BEB probabilities are greater.

\section{CONCLUDING REMARKS AND PERSPECTIVES}

We have investigated the hidden terminals scenario using 802.11. Our study have two goals: first, to propose a generic model in order to allow the study of different backoff algorithms and networks configuration. Second, based on this model, we provide a stochastic analysis of the hidden terminals scenario. This stochastic analysis allows us to derive a performance evaluation study that focuses on fairness issue. We introduce a metric $\left(\alpha_{i}\right)$ in order to compute short-term fairness. We show that the backoff stage distribution is not very sensitive to the access mechanism. The RTS/CTS access is unfair compared to basic access. We also show that the DIDD algorithm is more efficient and less fair than the BEB algorithm. Our results show that RTS/CTS mechanism solves the collision problem of hidden terminal but introduces a fairness problem.

Next work will be focused on the design of a backoff algorithms, for the hidden terminal scenario and for multi-hop network in general. An important goal is to design a new backoff algorithm where $\alpha_{i}$, the probability to be stopped, and the collision probability follow an appropriate function for a fair and efficient algorithm.

\section{REFERENCES}

[1] G. Bianchi. Performance analysis of the ieee 802.11 dcf. IEEE JSAC, 2000.

[2] P. Chatzimisios, A.C. Boucouvalas, V. Vitsas, A. Vafiadis, A. Oikonomidis, and P. Huang. A simple and effective backoff scheme for the ieee 802.11 mac protocol. In CITSA, Florida, USA, Jul 2005.

[3] C. Chaudet, D. Dhoutaut, and I. Guérin-Lassous. Performance issues with ieee 802.11 in ad hoc networking. IEEE Com. Mag., Aug 2005.
[4] C. Chaudet, I. Guérin Lassous, E. Thierry, and B. Gaujal. Study of the Impact of Asymmetry and Carrier Sense Mechanism in IEEE 802.11 Multihops Networks through a Basic Case. In PE-WASUN, Italy, Oct 2004.

[5] IEEE Standard for Information Technology. LAN and MAN - Specific Requirements - Part 11: Wireless LAN (MAC) and (Phy) Layer Specifications, 1997.

[6] M. Garetto, J. Shi, and E.-W. Knightly. Modeling media access in embedded two-flow topologies of multi-hop wireless networks. In Mobicom, Germany, Aug 2005.

[7] J. Hillston. A compositional approach to performance modelling. $\mathrm{PhD}$ thesis, The University of Edinburgh, United Kingdom, 1994.

[8] L. Kloul and F. Valois. Investigating unfairness scenarios in manet using 802.11b. In PE-WASUN, Canada, 2005.

[9] Z. Li, S. Nandi, and A. K. Gupta. Modeling the short-term unfairness of ieee 802.11 in presence of hidden terminals. In NETWORKING, Greece, pages 613-625, 2004. 
The 17th Annual IEEE International Symposium on Personal, Indoor and Mobile Radio Communications (PIMRC'06)

\section{APPENDIX}

The appendix illustrates how to model the RTS/CTS mechanism. It is necessary to modify both the transmitter-receiver model and medium one as it is noted in the following.

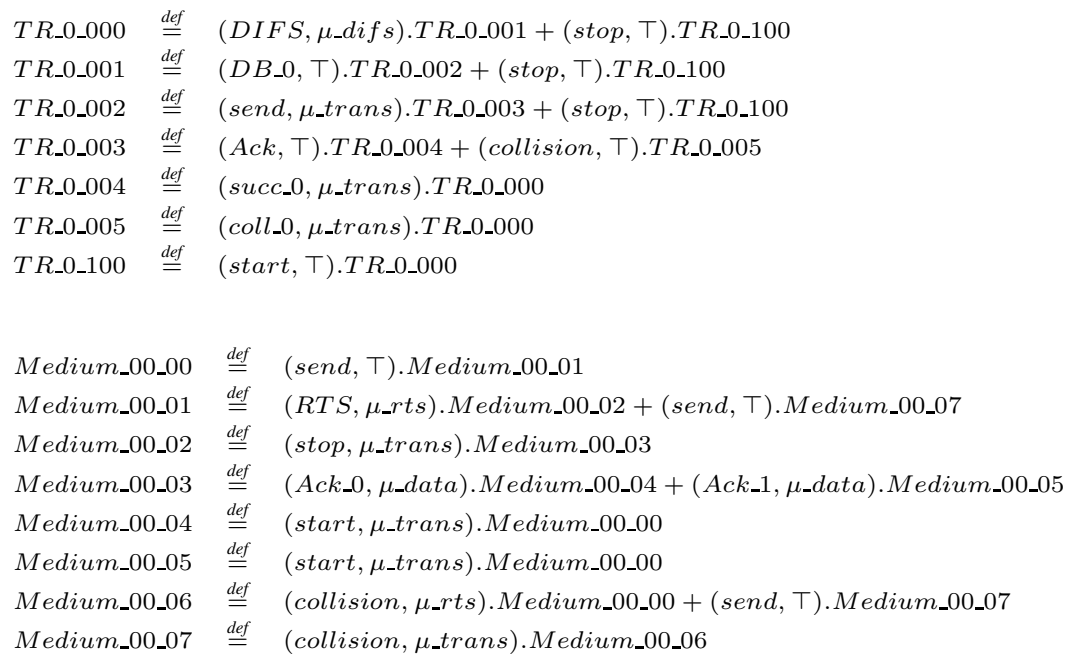

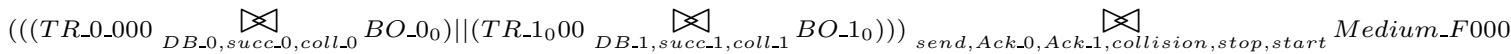

